\title{
3-SPS+RRS+PS Mechanism Analysis And Hessian Matrix Solution
}

\author{
Zhang-yuan \\ Key Laboratory for Computer Virtual Technology and \\ System Integration of Hebei Province, \\ Yanshan University, Qinhuangdao 066004,China. \\ Hebei Normal University of Science \& \\ Technology,Qinhuangdao Hebei 066004,China. \\ E-mail: wyzhang321@163.com

\section{Wangzhen} \\ Force Equipment Research Institute \\ Airlines,Beijing,100076,China. \\ E-mail: wangyuzhen@qq.com

\section{Guoansong} \\ China National Heavy Machinery Research Institute, \\ Xian 710032,China \\ E-mail: GUO Yansong@163.com
}

\author{
Guoi-juan \\ Key Laboratory for Computer Virtual Technology and \\ System Integration of Hebei Province, Yanshan \\ University, Qinhuangdao 066004,China. \\ E-mail:xjguo@ysu.edu.cn
}

\section{XiFeng-feng}

Department of Aerospace Engineering of Ryerson

University in Toronto, Canada.

\begin{abstract}
SPS+RRS+PS is a new type of mechanism. There is good application prospect in the field of aerospace. Especially some key kinetic characteristic calculation algorithms are implemented, which makes its calculation mechanized, thus it will contribute to make the dynamics performance of this mechanism optimized and ascended. In the paper, firstly, the degree of freedom is calculated and freedom nature is analyzed. Then the reverse solution is calculated, started with the nature of degree of freedom. And the mechanism velocity and acceleration inverse solutions are calculated in the direct derivation method. Although there are shortcomings that calculation process is complicated in direct derivation method, its calculation process is easy to realize mechanization. Then a kind of fast numerical calculation algorithm is given and MATLAB program is implemented. The algorithm has universal applicability to the mechanism of continuous differentiable inverse equation, and can be applied to general parallel mechanism.
\end{abstract}

Keywords: analysis of degree of freedom (DOF); position inverse solution; velocity and acceleration inverse solution*

\section{INTRODUCTION}

3-SPS+RRS+PS is a new type of mechanism, widely used in aviation field to control flight pose, and has a high research value. Experts and scholars have conducted in-depth research on it.

In 2009 the dynamics performance index of similar mechanism 3-RSS+PS+RRS was analyzed in [1]. In 2010 the dynamic performance of 3-SPS+1-RRS+1-PS mechanism was analyzed in [2].In 2012, kinematics performance and working space of a outer ring 3-SPS+1-PRS+1-PS and inner ring 3-SPS+1-RPS+1-PS mechanism were analyzed that is similar to the mechanism in [3].in 2013, the kinematic performance of 2-RRS-6SPS-1-PS mechanism was analyzed in [4].

From these studies above, we find that the two parallel mechanisms are similar and their construction types can both result in 3-SPS+RRS+PS all. Therefore,

\footnotetext{
* The National Natural Science Foundation of China (51175446)
} 
the research and analysis of 3-SPS+RRS+PS mechanism is the key of the study on this kind of hybrid mechanism. In 2013, Zhang Dayu's paper was published on the synthesis of mechanism [5].

The mechanism performance index and Hessian matrix are also studied deeply. In 2002, the dynamic performance index of the six degree of freedom(DOF) parallel mechanism and a less DOF parallel mechanism was proposed in [6], research and analysis has focused on the dynamics performance index in the following more than ten years, and based on Jacobian and Hessian matrix, meanwhile parallel and serial mechanism dynamics performance index system has been given systematically in [7]-[23]. According to the study of more than ten years, there are many affected factors in the mechanism dynamic performance. Among these mechanisms, two order influence coefficient Hessian matrix is a key element in [16]. Although, there are many good calculation methods of Hessian matrix, but the calculation process is so complex that it brings a lot of inconvenience to index calculation [17-19].

In this paper, the mechanism DOF is analyzed firstly, then the nature of the mechanism DOF is distinguished, and the position inverse solution is analyzed; and based on the results of the position inverse solution's results, the velocity inverse solution and acceleration inverse solution are calculated by direct derivation method. Although, there are a lot of very good methods to solve the mechanism velocity and acceleration in [20-22], direct derivation method is often described as complex calculation process in [23], but the simple ideas. For this we give a fast numerical calculating algorithm and have the above algorithm completed by MATLAB procedures. The algorithm has general applicability to continuously differentiable inverse equation mechanism, and is applied to general parallel mechanism. In addition, the Hessian matrix is directly separated from the inverse equation in the calculation process, which solves the difficulty of the Hessian matrix solution. In the analysis, we consider not only the correctness and effectiveness of the method, but also can mechanize the calculation. So not only the current requirements is met, but also Hessian matrix solution analysis can be automated and mechanized, and the automatic calculation basis is provided for future mechanism performance index analysis.

\section{THE DESCRIPTION OF MECHANISM AND THE}

\section{ESTABLISHMENT OF A COORDINATE SYSTEM}

Mechanism is composed of 1 RRS branched chain, 1 PS and 3 SPS branched chains, shown in Figure 1.

A coordinate system is established on each of the upper platform and the lower platform of the mechanism. As shown in Figure 2, the platform plane that is connected by each branched chain with the lower platform plane is XY plane, and fifth branched chain is $\mathrm{Z}$ axis to establish a fixed coordinate system O-XYZ. The X axis is through the first branched chain rotating pair, and the $\mathrm{Y}$ axis is through the second branched chain spherical joint. $\mathrm{Y}$ axis is parallel with the rotating pair by which the first branched chain is connected with the lower platform. On condition that the above platform central point $\mathrm{P}$ as the original point, the above platform normal as $z_{n}$ axis, the first branched spherical joint as $x_{n}$ axis and the second branched spherical joint as $y_{n}$ axis, the moving coordinate system $o_{n}-x_{n} y_{n} z_{n}$ is established. At the initial time, Each axis of moving coordinate system $o_{n}-x_{n} y_{n} z_{n}$ is parallel to each corresponding axe of O-XYZ..

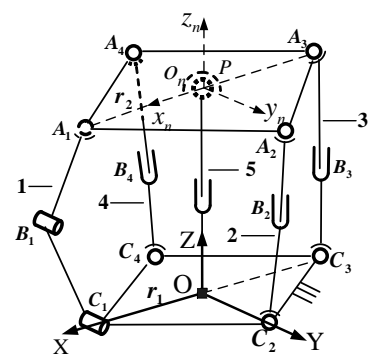

Figure 1. The establishment of a coordinate system

The rotating pair connecting the first branched chain with the lower platform is denoted by $C_{1}$, the spherical joint connecting with the upper platform is denoted by $A_{1}$, the rotating pair is denoted by $B_{1}$ in the middle part. The spherical joint connecting the 2,3 and 4 branched chain with the lower platform is denoted by $C_{i}(i=2,3,4)$, spherical joint connecting with the upper platform is 
denoted by $A_{i}(i=2,3,4)$, and the shifting pair is denoted by $B_{i}(i=2,3,4)$ in the middle part. $A_{i}(i=1,2,3,4)$ and $\mathrm{P}$ are coplanar, and distributed in the four equal-part points of a circle of which $\mathrm{P}$ is the center and $r_{2}$ is the radius $C_{i}(i=1,2,3,4)$ and $\mathrm{O}$ points are coplanar, and distributed in the four equal-part points of a circle of which $\mathrm{O}$ point is the center and $r_{1}$ is the radius. Upper platform turning

\section{DOF ANALYSES}

For the first branched chain as shown in Figure 2, C1 kinematic screw is $\$_{11}$, B1kinematic screw is $\$_{12}$, A1 kinematic screw is $\$_{13}, \$_{14}, \$_{15}$

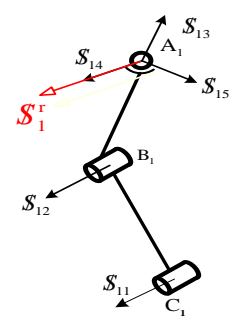

Figure 2. The first branched chain

By the geometric relationship between the screw and reciprocal screw, the reciprocal screw must pass the intersection of $\$_{13}, \$_{14}$ and $\$_{15}$, and parallel $\$_{12}$ and $\$_{11}$. Thus, reciprocal screw $\$_{1}^{\mathrm{r}}$ as shown in Figure 3, it passes A1 point and parallels $\$_{12}$ and $\$_{11}$, and so parallels $\mathrm{Y}$ axis in the fixed platform.

All chains from the second branched chain to the forth branched chain are SPS branched chains, which are common 6 DOF branched chains, unconstrained, so this paper will not discuss them.

For the fifth branched chain as shown in Figure 3, the shifting pair's motion screw is $\$_{51}$, and the spherical joint motion screw is $\$_{52}, \$_{53}$ and $\$_{54}$.

Due to the geometric relationship of the screw and the reciprocal screw, the reciprocal screw must pass the angle around $y_{n}$ axis is set to $\beta$ firstly, and turning angle around $x_{n}$ is $\alpha$ after rotating, then to move a distance of $P_{z}$ along $\mathrm{z}$ axis. At the initial time, $\alpha=0, \beta=0, \mathrm{p}$ point coordinate on mechanism upper platform is $(0,0, \mathrm{~h})$ in the coordinate system $\mathrm{O}-\mathrm{XYZ}, \mathrm{h}$ is the initial distance between $\mathrm{P}$ and $\mathrm{O}$, as shown in figure 1 .

intersection point of $\$_{52}, \$_{53}$ and $\$_{54}$, and is vertical with $\$_{51}$, so the two reciprocal screw $\$_{1}^{\mathrm{r}}$ and $\$_{2}^{\mathrm{r}}$ are parallel with $\mathrm{X}$ axis, $\mathrm{Y}$ axis in the fixed platform respectively.

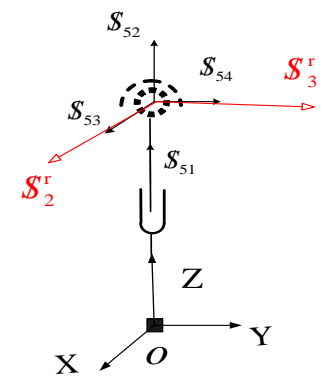

Figure 3. The fifth branched chain

The reciprocal screw of each branched chain analysis is analyzed among overall mechanism, as shown in figure 4. $\boldsymbol{S}_{2}^{\mathrm{r}}$ and $\boldsymbol{S}_{3}^{\mathrm{r}}$ are parallel to the $\mathrm{X}$ axis, $\mathrm{Y}$ axis in fixed platform constantly, so the non-special position type is not coincidence with the moving platform $O_{n} A_{1}$ and $O_{n} A_{2}$

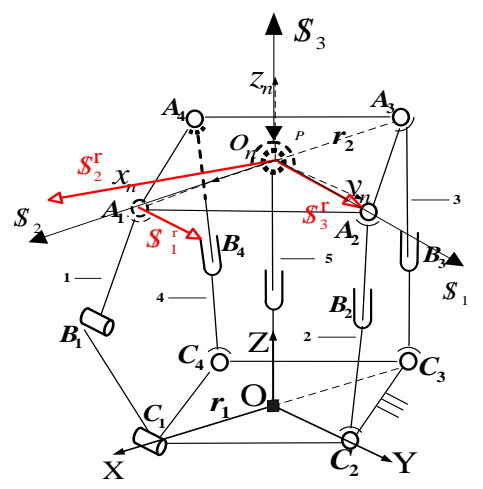

Figure 4. The DOF picture of mechanism 
As shown in Figure 4, because $\boldsymbol{S}_{1}^{\mathrm{r}}, \boldsymbol{S}_{2}^{\mathrm{r}}$ and $\boldsymbol{S}_{3}^{\mathrm{r}}$ are parallel or vertical, so they are linearly independent, so the mechanism is 3 DOF.

\section{INVERSE SOLUTION OF POSITION}

As shown in Figure 4, at initial time the coordinate each point that each branch chain connected to upper platform is:

$$
\left\{\begin{array}{l}
a_{1}=\left(a_{1 x}, a_{1 y}, a_{1 z}\right)=\left(r_{2}, 0,0\right) \\
a_{2}=\left(a_{2 x}, a_{2 y}, a_{2 z}\right)=\left(0, r_{2}, 0\right) \\
a_{3}=\left(a_{3 x}, a_{3 y}, a_{3 z}\right)=\left(-r_{2}, 0,0\right) \\
a_{4}=\left(a_{4 x}, a_{4 y}, a_{4 z}\right)=\left(0,-r_{2}, 0\right)
\end{array}\right.
$$

At initial time the coordinate each point that each chain branch chain connected to lower platform is:

$$
\left\{\begin{array}{l}
C_{1}=\left(C_{1 x}, C_{1 y}, C_{1 z}\right)=\left(r_{1}, 0,0\right) \\
C_{2}=\left(C_{2 x}, C_{2 y}, C_{2 z}\right)=\left(0, r_{1}, 0\right) \\
C_{3}=\left(C_{3 x}, C_{3 y}, C_{3 z}\right)=\left(-r_{1}, 0,0\right) \\
C_{4}=\left(C_{4 x}, C_{4 y}, C_{4 z}\right)=\left(0,-r_{1}, 0\right)
\end{array}\right.
$$

After the upper platform has been rotated and translated, The transformational matrix of the pose from beginning to end can be expressed by to $\mathrm{T}$ :

$$
\boldsymbol{T}=\boldsymbol{T}_{z} \boldsymbol{T}_{\alpha} \boldsymbol{T}_{\beta}=\left(\begin{array}{cc} 
& 0 \\
\boldsymbol{R}(\alpha, \beta) & 0 \\
& P_{z} \\
0 & 1
\end{array}\right)
$$

Among above:

$$
\boldsymbol{R}(\alpha, \beta)=\left(\begin{array}{ccc}
\cos \beta & \sin \alpha \sin \beta & \cos \alpha \sin \beta \\
0 & \cos \alpha & -\sin \alpha \\
-\sin \beta & \sin \alpha \cos \beta & \cos \beta \cos \alpha
\end{array}\right)
$$

After the upper platform pose being changed, The coordinate of $A_{i}(i=2,3,4)$ point in the 2,3 and 4 branch chain is:

$$
\left(\begin{array}{c}
A_{i x} \\
A_{i y} \\
A_{i z} \\
1
\end{array}\right)=\boldsymbol{T}_{S} \boldsymbol{R}(\alpha, \beta)\left(\begin{array}{c}
a_{i x} \\
a_{i y} \\
a_{i z} \\
1
\end{array}\right)
$$

In the formula, when $\boldsymbol{T}_{S}$ is at the initial position, due to the height of $\mathrm{Z}$ axis in the fixed coordinate system for the moving coordinate system caused by the spherical joint of the fifth branched chain, the homogeneous transformation matrix is :

$$
\boldsymbol{T}_{S}=\left(\begin{array}{llll}
1 & 0 & 0 & 0 \\
0 & 1 & 0 & 0 \\
0 & 0 & 1 & h \\
0 & 0 & 0 & 1
\end{array}\right)
$$

Through the above analysis, the 3 drives' (2, 3 and 4 branched chains) stem length vector $\boldsymbol{l}_{i}(i=2,3,4)$ in the fixed coordinate system is expressed as:

$$
\boldsymbol{L}_{i}=\boldsymbol{A}_{i}-\boldsymbol{C}_{i}
$$

Thereby the position inverse solution calculation equation is got

$$
L_{i}=\sqrt{L_{i x}^{2}+L_{i y}^{2}+L_{i z}^{2}}
$$

On the basis of the above algorithm, according to the mechanism different dimension parameters, a program is written to calculate the curve line inverse solution of the mechanism, as shown in figure 5:

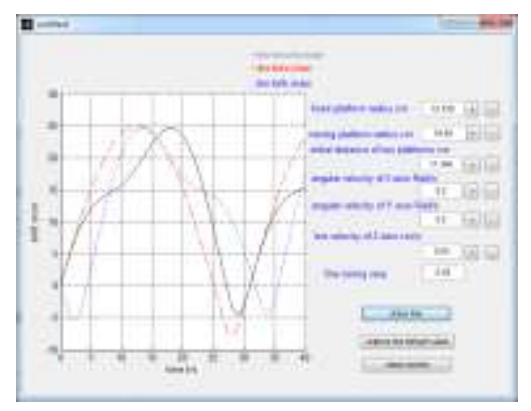

Figure 5. the curve line inverse solution

\section{HE INVERSE SOLUTION CALCULATION ABOUT VELOCITY AND ACCELERATION}

\section{A. formula derivation}

There are some methods to calculate the velocity and acceleration of parallel mechanism, including the influence coefficient method [18], differential method [2] etc. This paper adopts direct derivation method.

Three stem length equation $L_{2}, L_{3}$ and $L_{4}$ can be gotten by the mechanism inverse equation. They are the equation about $\alpha, \beta, h \alpha, \beta$ are rotating angle (two rotational DOF), and $h$ is the upper and lower shift(a translational DOF). 
Each $L$ can be denoted as $L_{i}(\alpha, \beta, h)$, or abbreviated as $L_{i}$.

$$
\text { Let } F=\left[\begin{array}{l}
L_{2}(\alpha, \beta, h) \\
L_{3}(\alpha, \beta, h) \\
L_{4}(\alpha, \beta, h)
\end{array}\right]
$$

\section{1) The velocity inverse solution}

The derivation to formula (9) and all rows can be expressed as:

$$
\begin{gathered}
{\left[\begin{array}{c}
\dot{L}_{2} \\
\dot{L}_{3} \\
\dot{L}_{4}
\end{array}\right]=\left[\begin{array}{lll}
\frac{\partial L_{2}}{\partial \alpha} & \frac{\partial L_{2}}{\partial \beta} & \frac{\partial L_{2}}{\partial h} \\
\frac{\partial L_{3}}{\partial \alpha} & \frac{\partial L_{3}}{\partial \beta} & \frac{\partial L_{3}}{\partial h} \\
\frac{\partial L_{4}}{\partial \alpha} & \frac{\partial L_{4}}{\partial \beta} & \frac{\partial L_{4}}{\partial h}
\end{array}\right] \cdot\left[\begin{array}{c}
\frac{\partial \alpha}{\partial t} \\
\frac{\partial \beta}{\partial t} \\
\frac{\partial h}{\partial t}
\end{array}\right]} \\
\operatorname{Let}\left[\tilde{G}_{i}\right]=\left[\begin{array}{lll}
\frac{\partial L_{i}}{\partial \alpha} & \frac{\partial L_{i}}{\partial \beta} & \frac{\partial L_{i}}{\partial h}
\end{array}\right] \text {, so formula (10) can }
\end{gathered}
$$

be expressed as:

$$
\left[\begin{array}{c}
\dot{L}_{2} \\
\dot{L}_{3} \\
\dot{L}_{4}
\end{array}\right]=[\tilde{G}] \cdot\left[\begin{array}{c}
\dot{\alpha} \\
\dot{\beta} \\
\dot{h}
\end{array}\right]
$$

In it, $[\tilde{G}]$ is the Jacobian matrix. In this paper, as $[\tilde{G}]$ is obtained from the inverse solution, $\tilde{G}$ is used to be distinguished from deriving from the normal solution equations in the related literature [18, 24-25].

2) Acceleration inverse solution

Acceleration inverse solution is the derivation to formula (11):

$$
\left[\begin{array}{c}
\ddot{L}_{2} \\
\ddot{L}_{3} \\
\ddot{L}_{4}
\end{array}\right]=\frac{\partial[\tilde{G}]}{\partial t} \cdot\left[\begin{array}{c}
\dot{\alpha} \\
\dot{\beta} \\
\dot{h}
\end{array}\right]+[\tilde{G}] \cdot\left[\begin{array}{c}
\ddot{\alpha} \\
\ddot{\beta} \\
\ddot{h}
\end{array}\right]
$$

And all stems' acceleration can be expressed as:

$$
\begin{gathered}
{\left[\begin{array}{c}
\ddot{L}_{2} \\
\ddot{L}_{3} \\
\ddot{L}_{4}
\end{array}\right]=\left[\begin{array}{lll}
\dot{\alpha} & \dot{\beta} & \dot{h}
\end{array}\right]\left[\begin{array}{l}
{\left[\tilde{H}_{2}\right]} \\
{\left[\tilde{H}_{3}\right]} \\
{\left[\tilde{H}_{4}\right]}
\end{array}\right]\left[\begin{array}{c}
\dot{\alpha} \\
\dot{\beta} \\
\dot{h}
\end{array}\right]+\left[\begin{array}{c}
\tilde{G}
\end{array}\right]\left[\begin{array}{c}
\ddot{\alpha} \\
\ddot{\beta} \\
\ddot{h}
\end{array}\right]} \\
=\left[\begin{array}{lll}
\dot{\alpha} & \dot{\beta} & \dot{h}
\end{array}\right]\left[\begin{array}{c}
\tilde{H}
\end{array}\right]\left[\begin{array}{c}
\dot{\alpha} \\
\dot{\beta} \\
\dot{h}
\end{array}\right]+\left[\begin{array}{c}
\tilde{G}
\end{array}\right]\left[\begin{array}{c}
\ddot{\alpha} \\
\ddot{\beta} \\
\ddot{h}
\end{array}\right]
\end{gathered}
$$

Among them:

$$
[\tilde{H}]=\left[\begin{array}{c}
\tilde{H}_{2} \\
\tilde{H}_{3} \\
\tilde{H}_{4}
\end{array}\right],\left[\tilde{H}_{i}\right]=\left[\begin{array}{ccc}
\frac{\partial^{2} L_{i}}{\partial \alpha^{2}} & \frac{\partial^{2} L_{i}}{\partial \alpha \partial \beta} & \frac{\partial^{2} L_{i}}{\partial \alpha \partial h} \\
\frac{\partial^{2} L_{i}}{\partial \beta \partial \alpha} & \frac{\partial^{2} L_{i}}{\partial \beta^{2}} & \frac{\partial^{2} L_{i}}{\partial \beta \partial h} \\
\frac{\partial^{2} L_{i}}{\partial h \partial \alpha} & \frac{\partial^{2} L_{i}}{\partial h \partial \beta} & \frac{\partial^{2} L_{i}}{\partial h^{2}}
\end{array}\right]
$$

\section{B. MATLAB calculation}

Realization method: The function Jacobian of MATLAB is called to get solution of $[\tilde{G}]$. Among it, $v=\left[\begin{array}{l}\alpha \\ \beta \\ h\end{array}\right],[\tilde{G}]$ is $3 \times 3$ matrix.

According to the calculating method in this paper, a algorithm to get $[\tilde{H}]$ is:

1) Input parameters: $L$ is a function vector ready to solve, and vector $V$ is composed of parameters to solve derivative.

2) Output: $H$ is the output Hessian matrix

3) Steps of the algorithm:

a) The Jacobian matrix of $L$ about $v$ is $[\tilde{G}]$ of last section

b) Get $m$, the number of the rows of $G$, and $n$, the number of the column.

c) On the each line of $G$ (i.e., $\dot{L}_{i}$ of above section), the partial differential coefficient of $\frac{\partial\left(\dot{L}_{i}\right)}{\partial t}$ is calculated and simplified.

d) Then the generated $n$ line matrix results is stored in line $i \times n-(n-1)$ to the line $i \times n$ of the output matrix $H$.

The calculation example:

$$
\text { For }\left[\begin{array}{lll}
\dot{\alpha} & \dot{\beta} & \dot{h}
\end{array}\right]=\left[\begin{array}{lll}
0.2 & 0.2 & 0.2
\end{array}\right]^{\prime},\left[\begin{array}{lll}
\ddot{\alpha} & \ddot{\beta} & \ddot{h}
\end{array}\right]
$$
$=\left[\begin{array}{lll}0 & 0 & 0\end{array}\right]^{\prime}$, the unit of the angular velocity is $\mathrm{rad} / \mathrm{s}$, and the unit of displacement is $\mathrm{cm}$. 


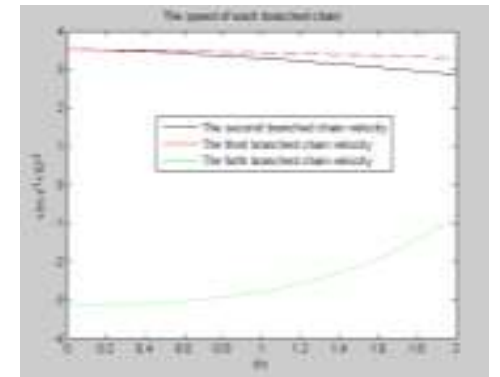

Figure 6. The branched chain velocity curve.

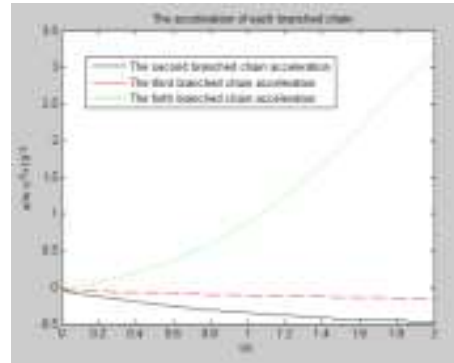

Figure 7. The branched chain acceleration curve

Note: this method only has general applicability to the mechanism of continuously differentiable inverse solution equation

\section{CONCLUSION}

(1) The new thinking of DOF calculation is used to analyze the DOF of the mechanism and gets the number 3, two rotational DOF and 1 mobile DOF. Among them one rotational DOF is the instantaneous DOF which needs special treatment in the inverse position solution calculation.

(2) The paper calculates the mechanism position inverse solution and the instantaneous DOF rotation transformation matrix, and realized it by rotational synthetic of the whole DOF, so as to ensure the position inverse solution is effective to any position type.

(3) By direct derivation of the inverse position solution equation, mechanism velocity and acceleration is calculated. That gives derivation of the principle, and also a fast MATLAB calculation method is given based on MATLAB. This method, only using the inverse solution equation, can quickly and automatically get the Hessian matrix solution, and the problem of the Hessian matrix computing intensively and large amounts of calculation, providing a lot of convenience to calculate performance indicators.

\section{REFERENCE}

[1] Guo X J, Zhang Q, Du X et al. Analysis for Dynamics Performance Indices of 3-RSS+PS+RRS Parallel Mechanism[J]. ICIC Express Letters, 2009, 3(3):793-798.
[2] YANG Yulin, FAN Shaoshuai, DU Xiong, GUO Xijuan. Analysis on Size Optimization of Equivalent Parallel Mechanism of Stationary Ring of Swashplate [J]. Chinese Journal of Mechanical Engineering, 2010,46 (7):48-55

[3] Chang Fuqing, Jia Haixin, Zhang Dayu, Guo Xijuan. Kinematic Analysis of a New Nested Inner-outer Ring Mechanism[J]. ICIC Express Letters, 2012, 3(6):1-ELB12-0450.

[4] GUO Xijuan, HUANG Tianyu, CHANG Fuqing. Kinematics of a New Hybrid Mechanism with Reduced DOF and Nested Dynamic Connection, Chinese Journal of Mechanical Engineering, 2013,49(1):1-7.

[5] Xijuan Guo, Dayu Zhang, Qingjia Geng, et al. The synthesis of dynamic junction over-constrained parallel-parallel mechanism based on the weighted directed graph theory [J]. ICIC Express Letters,2013,7(11):3057-3065.

[6] GUO Xijuan. A review about basic theory of mechanism Dynamics for parallel robotics. Yanshan University doctoral dissertation of science in engineering.

[7] Huang Zhen Guo Xijuan. Provement of The Correctness of Virtual Mechanism Method[J]. Chinese Journal of Mechanical Engineering, 2001, 37(5):40-43.

[8] Guo xijuan. The Kinematics Performance of Plane Parallel Mechanism 3RRR Analysis. Robot Technique and Application, 2002,(4): 41-45

[9] Yu Meisheng, Zhu Xijun, Guo Xijuan. Base on The RSSR-SCs Patial Mechanisms Velocity and Acceleration Performance Parameters Analysis, 2002,(3):26-31.

[10] Guo X J, LIU S. Analysis for dynamics performance indices of 4-RR(RR)R parallel mechanism[J]. International Journal of Innovative Computing Information and Control, 2006, 2(4): 849-862.

[11] G.Qingjia, G.Xijuan. Research on Acceleration Performance Indices for Serial Mechanism. IEEE 2007 International conference on Automation and Logistics (ICAL), 2007,8; 2160-2164.

[12] Guo Xijuan, Peng Yanmin, Geng Qingjia. Dynamics Performance Analysis for LR-Mate Robot. Chinese Journal of Mechanical Engineering, 2007.11:84-87.

[13] Geng Q J, Guo X J . Research on acceleration performance indices for serial mechanism[C]. 2007 IEEE ICAL, Shandong, Jinan.

[14] Zhang Weiwei, Guo Xijuan. Dynamic Performance Analysis and Simulation of Stanford Robot. Computer Simulation, 2007,24(5):138-141.

[15] Guo Xijuan, Geng Qingjia. Analysis for Acceleration Performance Indices of Serial Robots. Chinese Journal of Mechanical Engineering, 2008; 44(9)56-60.

[16] Guo Xijuan. Mechanism Performance Indices Analysis and simulation (monograph)[M]. Science Press. 2010.

[17] Huang zhen, Liu Jingfang, Li Yanwen. On the Mechanism Degrees of Freedom -- The General Formula of Degree of Freedom Search for 150 Years[M].Beijing: Science Press, 2011

[18] Huang zhen, Zhao Yongsheng, Zhao Tieshi. Advanced Spatial Mechanism [M].piking: Higher Education Press, 2006.

[19] Huang Zhen, Kong Lingfu, Fang Yuefa. The Parallel Robot Mechanism Theory and Its control [M]. beijing: China Machine Press, 1997.

[20] Zhao Jingshan, Feng Zhijing, Zhu Fulei. Robot Mechanism Analysis Theory of Degree of Freedom [M]. 2009.

[21] LIU Shanzeng, YU Yueqing, LIU Qingbo, SU Liying, SI Guoning. Dynamic Analysis of 3-RRC Parallel Manipulator [J], Chinese Journal of Mechanical Engineering, 2009, 45 (5):220-224

[22] Feng Zhiyou, Zhang Yan, Yang Tingli, Zhang Ce. Inverse Dynamics of a 2UPS-2RPS Parallel Mechanism by Newton-Euler Formulation [J]. Transactions of The Chinese Society for Agricultural Machinery, 2009, 40 (4):193-197.

[23] Sun Lining, YuHui, Zhu Yuhong, Zhang Xiufeng. Rearch on Influence Coefficient of Mechanism and Jaeobia Matrix of Parallel Robot [J].Journal of Harbin Institute of Technology, 2002 34(6):810-814. 
[24] YANG Yulin, HUANG Shijun, LIU Xiping, GUO Xijuan. Analysis of Dynamic Performance of 2-RUUS Mechanism. Chinese Journal of Mechanical Engineering, 2009, 45(11):1-9.

[25] LI Sh-i hua, HUANG Zhen. Kinematical Analysis for a Special 3-UPU Truncated Pyramid Parallel Mechanism [J]. Machine Design, 2005, 7(22):16-19. 